\title{
Evaluation of Business-Oriented Performance Metrics in e-Commerce using Web-based Simulation
}

\section{Mitrevski, Pece ${ }^{a}$ and Hristoski, Ilija ${ }^{b}$}

${ }^{a}$ Faculty of Information and Communication Technologies - Bitola, "St. Kliment Ohridski" University, Republic of Macedonia, 'Faculty of Economics - Prilep, "St. Kliment Ohridski" University, Republic of Macedonia.

\begin{abstract}
The Web 2.0 paradigm has radically changed the way businesses are run all around the world. Moreover, e-Commerce has overcome in daily shopping activities. For management teams, the assessment, evaluation, and forecasting of online incomes and other business-oriented performance measures have become 'a holy grail', the ultimate question imposing their current and future e-Commerce projects. Within the paper, we describe the development of a Web-based simulation model, suitable for their estimation, taking into account multiple operation profiles and scenarios. Specifically, we put focus on introducing specific classes of e-Customers, as well as the workload characterization of an arbitrary e-Commerce website. On the other hand, we employ and embed the principles of the system thinking approach and the system dynamics into the proposed solution. As a result, a complete simulation model has been developed, available online. The model, which includes numerous adjustable input variables, can be successfully utilized in making 'what-if'-like insights into a plethora of business-oriented performance metrics for an arbitrary e-Commerce website. This project is, also, a great example of the power delivered by InsightMaker ${ }^{\circledR}$, free-ofcharge Web-based software, suitable for a collaborative online development of models following the systems thinking paradigm.
\end{abstract}

Keywords: e-Commerce; business-oriented performance metrics; evaluation; Web-based simulation; system dynamics. 